\title{
Hemochromatosis Gene Mutation and Erythrocytosis
}

\section{Judit Várkonyi*}

Department of Internal Medicine, Semmelweis University, Hungary

*Corresponding author: Judit Várkonyi, Department of Internal Medicine, Semmelweis University, Kútvölgyi út 4, Budapest, Hungary 1125; Email:

\section{Research Article}

Volume 2 Issue 2

Received Date: July 23, 2018

Published Date: July 31, 2018

DOI: $10.23880 / \mathrm{hij}-16000126$

varkonyi.judit@med.semmelweis-univ.hu

\section{Abstract}

In a patient group presenting with either increased Hgb level or high RBC count HFE gene mutations occured in two-third of the patients. These patients had normal erythropoietin levels and had no JAK2 mutation. Out of the 22 patients, 12 had the $H 63 D$ mutation in heterozygous / homozygous or compound heterozygous forms, 2 had $C 282 Y$ mutation and 7 had wild type HFE gene. In contrast to the classical form of type I Hemochromatosis with C282Y homozygous mutation, characterised by high serum iron, transferrin saturation and ferritin levels, patients with the $H 63 D$ mutation had normal iron content. In the second part of the study authors analysed iron and red cell parameters of patients available from their Hemochromatosis registry. The results were similar: those who had the H63D mutation, had more red cells and less iron content. It seems like in those having this mutation more iron is consumed for the red blood cell production, but the explanation for this phenomenon is still missing. The role of $H 63 D$ mutation in this process should still be clarified.

Keywords: HFE; H63D; Erythrocytosis

Abbreviations: RBC: Red Blood Cell; JAK2: Janus Kinase 2; EPO: Erythropoietin; PV: Polycythaemia; HH: Hemochromatosis.

\section{Introduction}

Patients with high red blood cell count (RBC) and high hemoglobin concentration with janus kinase 2 (JAK2) mutation and low erythropoietin (EPO) level- represent the group of true polycythaemia (PV) and those with wt $J A K 2$ and high EPO are the group of the so-called secondary polycythaemia forms [1]. Those significant number of patients, however who presenting either high haemoglobin or RBC levels and has no JAK2 mutation, and has normal EPO levels still remain a diagnostic challenge.
It has been noticed, that regular therapeutic phlebotomy -as the main treatment option for Hereditary Hemochromatosis $(\mathrm{HH})$ patients- is well tolerated by them, meaning that anemia does not develop even in the first year of diagnosis, when $400 \mathrm{ml}$ blood / occasion is drained many times until the goal ferritin level achieved and maintained thereafter. In contrast to this, for a voluntary blood donor five blood donations are permitted in a year to avoid the development of anemia. In a survey of voluntary blood donors, the ratio of $H 63 D$ heterozygotes was $31 \%$ in the group of the so-called super donors who donate 30-107 occasions [2]. Whether the high iron availability of $\mathrm{HH}$ patients is enough to explain this extremely good compensation of blood loss? In this study authors discuss this problem based on literature data and their recent findings. 


\section{Materials and Methods}

The first study population consisted of $22(16 \mathrm{M} / 6 \mathrm{~F}$, mean age: 61,6 yrs) non selected, consecutive and naive subjects who had not phlebotomy or other therapy before, were not blood donors and the reason of enrollment was their high Hgb levels or RBC counts found on rutin laboratory analysis. They were JAK2 wt (confirmed also by gene sequence analysis) and had normal EPO levels.

The second study population consisted of 35 hemochromatosis patients (16 with homozygous $C 282 Y$,11 with compound heterozygous,- and 8 with homozygous $H 63 D$ mutation) from the Hemochromatosis patients registry of our institute. All were males. Female patient were excluded from this study because of their regular menstrual blood loss and also those males, who had known co-morbidities causing bleeding or bone marrow disorders with impaired red cell production, like myelodysplastic syndromes.
The participants signed informed consents, and the study was approved by the Institutional Ethics Committee. Whole genomic DNA was isolated from anticoagulated peripheral blood with Puregene Gentra DNA Isolation kit. HFE gene sequence variant C282Y (exon 4, c.1066G>A, substitution of Cys282 to Tyr, rs1800562); and $H 63 D$ (exon 2, c.408C $>$ G, substitution of His63 to Asp, rs1799945) were investigated by Light Cycler technology (Roche Diagnostics) applying melting curve analysis with the hybridization probe detection format [3]. The JAK2 gene V617F mutation (exon 12, c.1849G>T, substitution of Val617 to Phe) was detected by allele specific multiplex PCR [4].

\section{Results}

In the first study population serum iron, transferrin saturation and ferritin concentration did not differ from normal values, but red blood cell count / or hemoglobin was higher in those, who had in any type the HFE gene mutation Table 1.

\begin{tabular}{|c|c|c|c|c|c|c|}
\hline & $\mathrm{EPO}$ & $\mathrm{RBC}$ & $\mathrm{Hgb}$ & $\mathrm{Se} \mathrm{Fe}$ & Tf sat & Ferritin \\
\cline { 2 - 6 } & $\mathrm{mU} / \mathrm{ml}$ & $\mathrm{Tera} / \mathrm{l}$ & $\mathrm{g} / \mathrm{l}$ & $\mathrm{umol} / \mathrm{l}$ & $(\%)$ & $\mathrm{ug} / \mathrm{l}$ \\
\hline \multirow{2}{*}{ HFE wt $\mathrm{n}=7$} & 5,6 & 5,7 & 181 & 23,5 & 37 & 293 \\
\hline \multirow{2}{*}{ HFE mut $\mathrm{n}=15$} & 11,9 & 6,0 & 187 & 17,8 & 30 & 242 \\
\hline \multirow{2}{*}{ Normal } & \multirow{2}{*}{$2,6-18,5$} & $\mathrm{~F} / \mathrm{M} \max$ & $\mathrm{F} / \mathrm{M} \max$ & $\mathrm{F} / \mathrm{M} \max$ & \multirow{2}{*}{$16-45$} & $\mathrm{~F} / \mathrm{M} \max$ \\
\cline { 3 - 7 } & & $5,1 / 5,9$ & $153 / 175$ & $28 / 32,2$ & & \\
\cline { 4 - 7 } & & &
\end{tabular}

Mean values are shown. The upper normal values are given for Females and Males.

Table 1: Iron and red cell parameters of JAK2 wt patients with erythrocytosis having or having not HFE gene mutation.

Regarding HFE gene status the wild type/ mutant ratio was 7 / 15. When the type of HFE gene mutation was analysed, 2 patients had $C 282 Y$ and 8 were $H 63 D$

heterozygotes whereas 4 compound heterozygotes and 1 H63D homozygote were also found (Table 2).

\begin{tabular}{|c|c|c|c|c|}
\hline & $\begin{array}{c}C 282 Y \\
1 / 0 \\
\end{array}$ & $H 63 D$ 0/1 & $H 63 D 0 / 2$ & $\begin{array}{c}C 282 Y / H 63 D \\
01 / 01 \\
\end{array}$ \\
\hline HFE mutant (n:15) & 2 & 8 & 1 & 4 \\
\hline
\end{tabular}

Abbreviations: $1 / 0$ or $0 / 1$ are heterozygous, $0 / 2$ are homozygous, $01 / 01$ : compound heterozygous.

Table 2: HFE gene mutation results of the 15 patients with JAK2wt erythrocytosis.

In the second study population it was clearly seen that the highest red blood cell counts occurred in those who contain the $H 63 D$ mutation and the highest iron values in those, who had the C282Y in homozygous form Table 3. 


\section{Haematology International Journal}

\begin{tabular}{|c|c|c|c|c|c|}
\hline & RBC Terra/l & Hgb g/l & SeFe umol/l & tfsat (\%) & ferritin ug/l \\
\hline compound & 5,1 & 164 & 25,8 & 43 & 418 \\
n=11 & $(4,5-6,07)$ & $(140-188)$ & $(15,1-39,3)$ & $(23-69)$ & $(31-1678)$ \\
\hline H63D & 5,5 & 167 & 29,9 & 40 & 336 \\
n=8 & $(4,8-6,76)$ & $(158-183)$ & $(20,5-41,6)$ & $(30-48)$ & $(114-547)$ \\
\hline C282Y & 4,81 & 154 & 38,1 & 75 & 1259 \\
n=16 & $(4,3-5,75)$ & $(140-168)$ & $(27,5-53,9)$ & $(42-103)$ & $(450-2580)$ \\
\hline
\end{tabular}

Mean values are shown. The upper normal values are shown in Table 1.

Table 3: Iron and red cell parameters of a Hemochromatosis patient population.

\section{Discussion}

In this preliminary study $H F E$ gene mutation occurred in two-third of patients presenting either high Hgb or RBC count, but did not fulfil the criteria of PV. There was a male preponderance. Literature data confirm that $\mathrm{HH}$ genetic polymorphism is associated with more iron availability / and more iron absorption especially in a compound heterozygous form $[5,6]$. Would this be enough to explain erythrocytosis? More iron mean more red cell or Hgb? It has been shown that both types of TfRs bind to HFE proteins and have role in iron sensing [7]. TfR2 is recently found to be an EPO partner receptor [8]. Based on these findings we might explain erythrocytosis seen in Tfr2 knockout mice and in Type 3 hemochromatosis patients who tolerate well repeated phlebotomies. Authors of the present study therefore assumes that coexistent TfR2 gene mutation might hide in the background in JAK2wt/norm EPO / HFE - related or unrelated erythrocytosis, that is to be further analysed. Multiple pathways might exist influencing erythropoiesis other than EPO signaling mechanism. Among these could be- not surprisingly- genes participating in iron metabolism regulation that still should be explored.

\section{Conclusion}

The significant ratio of HFE gene mutation in the group of patients presenting with high red blood cell count and hemoglobin level outlines the importance to perform the test in all cases of unexplained (JAK $2 \mathrm{wt}$, normal level EPO) erythrocytosis.

Acknowledgements: Author is thankful to Ambrus Gángó, Richárd Kiss, Csaba Bödör, Dorottya Csuka and Márta Kókai for the genetic analysis.

\section{References}

1. Daniel Arber A, Orazi A, Hasserjian R, Thiele J, Borowitz MJ, et al. (2016) The 2016 revision to the
World Health Organization classification of myeloid neoplasms and acute leukemia. Blood 127(20): 23912405.

2. Andrikovics H, Kalmár L, Bors A, Fandl B, Petri I, et al. (2001) Genotype screening for Hereditary Hemochromatosis among Voluntary Blood Donors in Hungary. Blood Cells Mol Dis 27(1): 334-341.

3. Feder JN, Gnirke A, Thomas W, Tsushiashi Z, Ruddy DA, et al. (1996) A novel MHC class I -like gene is mutated in patients with hereditary haemochromatosis. Nat Genet 13(4): 399-408.

4. Baxter EJ, Scott LM, Campbell PJ, East C, Fourouclas N, et al. (2005) Acquired mutation of the tyrosine kinase $J A K 2$ in human myeloproliferative disorders. Lancet 365(9464): 1054-1061.

5. Hunt JR, Zeng H (2004) Iron absorption by heterozygous carriers of the HFE C282Y mutation associated with hemochromatosis. AM J Clin Nutr 80(4): 924-931.

6. Raddatz D, Legler T, Lynen R, Addicks N, Ramadori G (2003) HFE genotype and parameters of iron metabolism in German first-time blood donors evidence for an increased transferrin saturation in C282Y heterozygotes. Z Gastroenterol 41(11): 10691076.

7. Goswami T, Andrews NC (2006) Hereditary hemochromatosis protein, HFE interaction with transferrin receptor 2 suggests a molecular mechanism for mammalian iron sensing. J Biol Chem 281(31): 28494-28498.

8. Kawabata H, Germain RS, IkezoeT, Tong X, Green EM, et al. (2001) Regulation of expression of murine transferrin receptor 2. Blood 98(6): 1949-1954. 